\title{
Energia Eletromagnética na Superfície Terrestre: estimativa multitem- poral do albedo em Garanhuns-PE
}

\author{
Electromagnetic energy in Land area: multitemporal estimate of the albedo in Garanhuns-PE
}

Felippe Pessoa de Melo e Rosemeri Melo e Souza

${ }^{1}$ Doutorando, Programa de Pós-Graduação em Geografia - PPGEO, Universidade Federal de Sergipe, São Cristóvão, Brasil felippemelo@hotmail.com

${ }^{2}$ Doutora, Núcleo de Engenharia Ambiental - NEAM, Universidade Federal de Sergipe - UFS, São Cristóvão, Brasil

\begin{abstract}
Resumo
O presente trabalho teve como objetivo, realizar uma análise multitemporal do espectro eletromagnético, em Garanhuns-PE, a partir do albedo. Utilizando as geotecnologias do Sensoriamento Remoto e Sistemas de Informações Geográficas (SIG), mais os rasters do Instituto Nacional de Pesquisas Espaciais (INPE), foi possível estimar e cartografar as mudanças dos padrões de reflectâncias dos alvos. Ao passo que a poligonal urbana ampliouse, a capacidade de reflectância dos alvos também aumentou. Evidenciando, que nem sempre a maximização do albedo significa redução termal. Logo alguns objetos podem ter uma grande capacidade de reflexão, mas, a energia absorvida é conservada por mais tempo.
\end{abstract}

Palavras-chave: Sensoriamento Remoto, SIG, Energia Eletromagnética, Albedo.

\begin{abstract}
This study aimed to conduct a multi-temporal analysis of the electromagnetic spectrum, in Garanhuns-PE from the albedo. Using the geo of Remote Sensing and Geographic Information Systems (GIS), plus the rasters of the National Institute for Space Research (INPE), it was possible to estimate and map the changes in reflectance patterns of the targets. While urban polygon has expanded the ability of reflectance, targets also increased. Showing that not always the maximization of albedo means thermal reduction. Soon some objects can have a great capacity for reflection, but absorbed energy is conserved longer.
\end{abstract}

Keywords: Remote Sensing, GIS, Electromagnetic Energy, Albedo. 


\section{Introdução}

As transformações antrópicas realizadas na paisagem estão cada vez mais intensas e aceleradas. Existindo uma constante necessidade e demanda de novas áreas para expansão das poligonais urbanas e agrárias. Ambas as situações interferem de forma drástica no comportamento do espectro eletromagnético. Existindo uma forte tendência a redução da capacidade de reflectância e maximização do potencial de absorção. Esse fenômeno ocorre principalmente, devido à introdução de alvos que possuem albedos inferiores aos antecessores.

Entre as propriedades físicas dos objetos, destaca-se o albedo, normalmente dado em porcentagem, que se caracteriza pela capacidade que os corpos têm de refletir a radiação solar que incide sobre eles. O albedo varia de acordo com a cor e a constituição do corpo. Assim, será máximo nos corpos brancos e mínimo nos corpos pretos. (MENDONÇA; DANNI-OLIVEIRA, 2007).

A quantidade e qualidade da energia eletromagnética refletida e emitida pelos objetos terrestres resultam das interações entre a energia eletromagnética e estes objetos. Essas interações são denominadas pelas propriedades físico-químicas e biológicas desses objetos e podem ser identificadas nas imagens e nos dados de sensores remotos. Portanto, a energia eletromagnética refletida e emitida pelos objetos terrestres é à base de dados para todo o processo de sua identificação, pois ela permite quantificar a energia espectral refletida e/ou emitida por estes, e assim avaliar suas principais características. Logo os sensores remotos são ferramentas indispensáveis para a realização de inventários, de mapeamento e de monitoramento de recursos naturais. (GALVÍNCIO et al., 2006).

Conforme Silva et al. (2006), o saldo de radiação exerce um papel fundamental nos processos de troca de calor e massa na baixa troposfera, uma vez que se constitue no principal responsável pelo aquecimento do solo, do ar, e principalmente, pela evapotranspiração da vegetação nativa e das culturas. Para determinação do saldo de radiação, especialmente em escala regional, faz-se necessário o conhecimento do albedo, também muito importante em estudos de mudanças climáticas, desertificação, queimadas e meio ambiente em geral.

Nesse contexto as tecnologias do Sensoriamento Remoto e Sistemas de Informações Geográficas (SIG), servem de aparato geotecnológico, possibilitando a análise e compreensão da dinâmica superficial da paisagem. A primeira, fornecendo imagens via satélite (multitemporais), e a segunda a confecção de bancos de dados, com diversas camadas de informações passivas de serem interpoladas. Possibilitando reconstituir cenários pretéritos, mensurar/acompanhar as transformações que estão ocorrendo e modelar/estimar situações futuras.

Segundo Christofoletti (1999), a modelagem pode ser considerada como instrumento entre os procedimentos metodológicos da pesquisa científica. A justificativa está no fato de que a construção de modelos a respeito dos sistemas ambientais representa a expressão de uma hipótese científica, que necessita ser validada como sendo enunciado teórico sobre o sistema ambiental focalizado. Essa avaliação configura-se como teste de hipóteses. Sob esse viés, a construção de modelos pode ser considerada como sendo procedimento inerente à pesquisa científica e a sua elaboração deve ser realizada acompanhando os critérios e normas da metodologia científica.

Mas para que as análises tenham respaldo científico, faz-se necessário a utilização de uma chave de interpretação, caso esse procedimento não seja realizado, as avaliações não passarão de meras deduções, provenientes do senso comum.

As imagens obtidas por sensores remotos, independente do processo de formação, registram a energia proveniente dos alvos da superfície desejada. Independente da resolução e escala, as matrizes apresentam os elementos básicos para análise e interpretação, a partir dos quais se extraem informações de objetos, áreas ou fenômenos. Esses elementos ou variáveis são: tonalidade/ cor, textura, tamanho, forma, sombra, altura, padrão e localização. (FLORENZANO, 2011).

Sendo assim a presente pesquisa teve como objetivo, realizar uma análise multitemporal do comportamento do espectro eletromagnético, na superfície do município de Garanhuns-PE, tendo como referência o albedo.

\section{Caracterização da área}

\subsection{Localização}

O território pernambucano fica inserido na região Nordeste do Brasil, conforme a Figura 1. A qual possui uma área de $1.561 .092 \mathrm{~km}^{2}$, estando distribuída pelos estados de Alagoas $\left(27.807 \mathrm{~km}^{2}\right)$, Bahia $\left(566.355 \mathrm{~km}^{2}\right)$, Ceará (148.853 km²) Maranhão (335.861 km²), Paraíba (56.524 $\left.\mathrm{km}^{2}\right)$, Pernambuco $\left(98.192 \mathrm{~km}^{2}\right)$, Piauí $\left(252.718 \mathrm{~km}^{2}\right)$, Rio Grande do Norte $\left(52.867 \mathrm{~km}^{2}\right)$ e Sergipe $\left(21.914 \mathrm{~km}^{2}\right)$.

Pernambuco está subdividido em cinco mesorregiões, denominadas de Sertão $\left(37.934 \mathrm{~km}^{2}\right)$, São Francisco $\left(24.471 \mathrm{~km}^{2}\right)$, Agreste $\left(24.561 \mathrm{~km}^{2}\right)$, Mata $\left(8.427 \mathrm{~km}^{2}\right) \mathrm{e}$ Metropolitana de Recife $\left(2.798 \mathrm{~km}^{2}\right)$.

A mesorregião do Agreste desmembra-se nas microrregiões do Alto Capibaribe $\left(1.783 \mathrm{~km}^{2}\right)$, Brejo Pernambucano $\left(2.554 \mathrm{~km}^{2}\right)$, Garanhuns $\left(5.184 \mathrm{~km}^{2}\right)$, Médio Capibaribe $\left(1.763 \mathrm{~km}^{2}\right)$, Vale do Ipanema $\left(5.376 \mathrm{~km}^{2}\right)$ e Vale do Ipojuca $\left(7.899 \mathrm{~km}^{2}\right)$.

O gentílico de Garanhuns, delimita-se pelas coordenadas geográficas de $-8^{\circ} 51^{\prime} 37^{\prime \prime} /-8^{\circ} 55^{\prime} 40^{\prime \prime}$ e $-36^{\circ} 26^{\prime} 06^{\prime \prime}$ / $-36^{\circ} 30^{\prime} 52^{\prime \prime}$. O qual faz divisa com 11 municípios, como pode ser visto na Figura 2. Ao norte com os municípios de Capoeiras (7,3 km), Jucati (5,4 km); ao sul Correntes $(12,7 \mathrm{~km})$, Lagoa do Ouro (2,3 km), Brejão $(38,97 \mathrm{~km})$, Terezinha $(4,25 \mathrm{~km})$; a leste São João $(25,8 \mathrm{~km})$, Palmeirina $(3,4 \mathrm{~km})$; a oeste Saloá $(8,31 \mathrm{~km})$, Paranatama $(9,36$ 


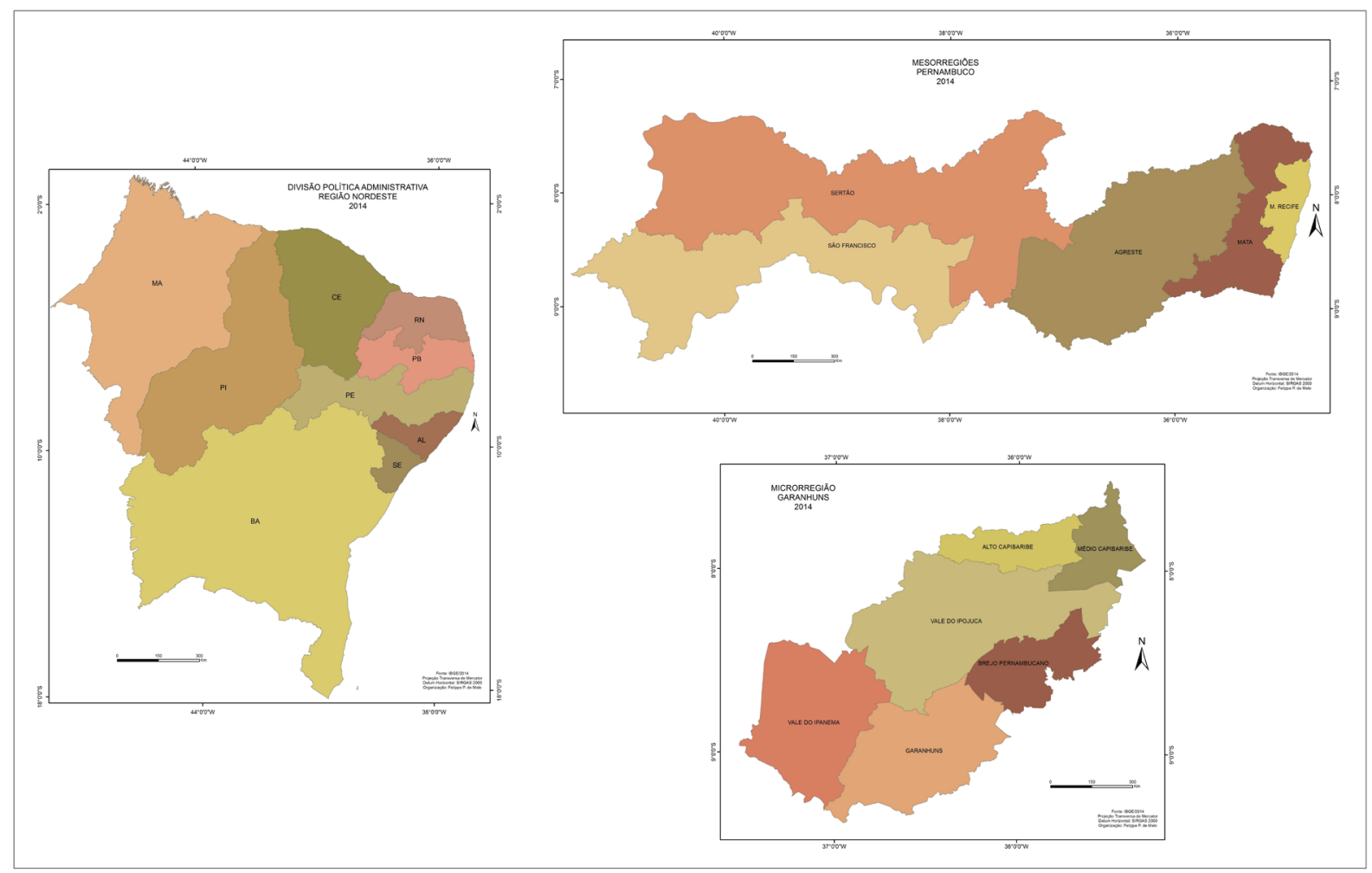

Figura 1 -Localização do estado de Pernambuco e suas subdivisões regionais Fonte: IBGE (2014)

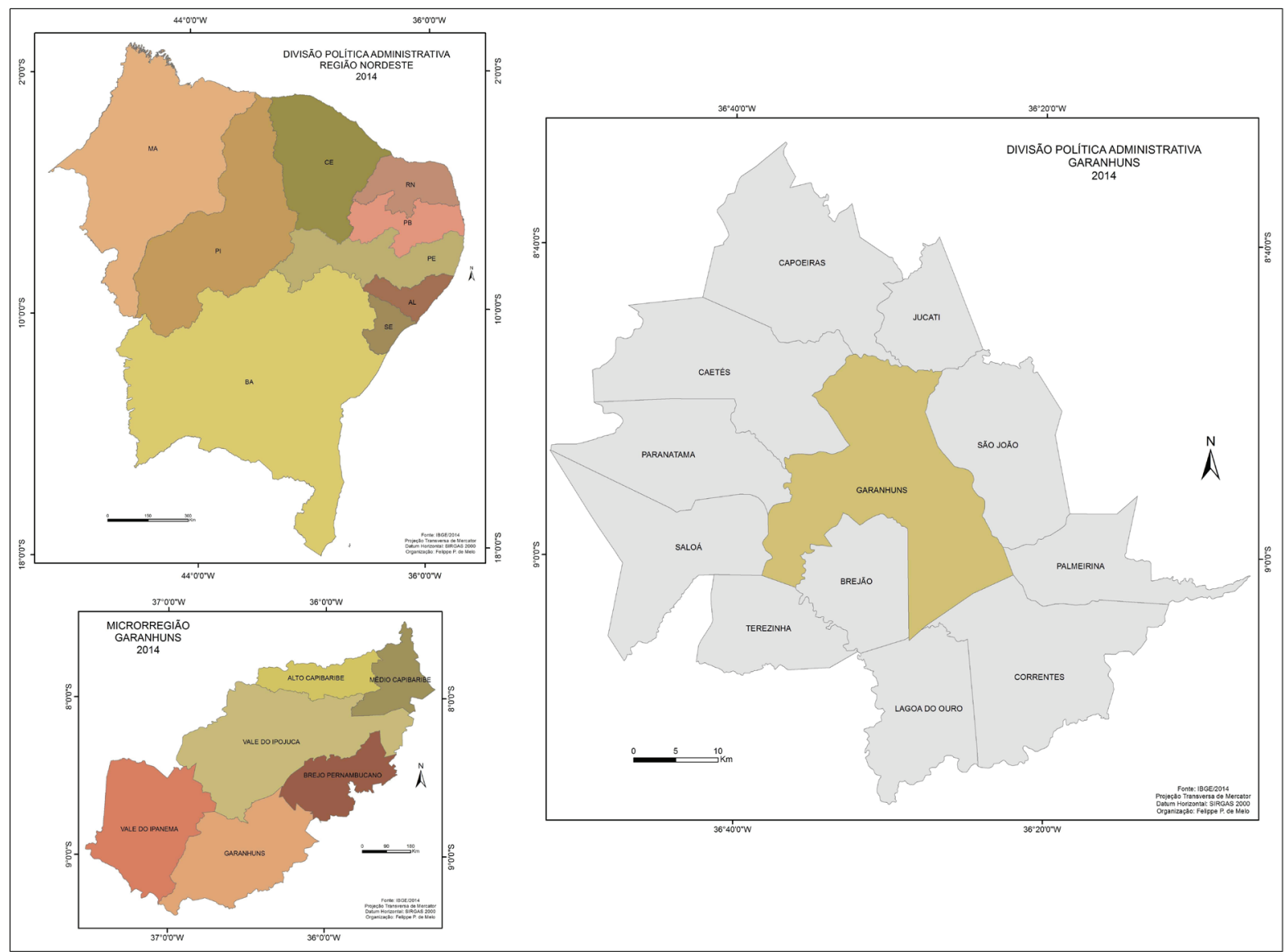

Figura 2 - Localização da área de estudo Fonte: IBGE (2014) 
$\mathrm{km})$, Caetés (25,16 km).

A referida cidade tem duas rotas principais para capital pernambucana. A primeira pela BR 101, percorrendo um trecho de $242 \mathrm{~km}$; e a segunda, passando por duas BRs $423 / 232$, percorrendo $80,6 \mathrm{~km}$ pela 423 até São Caetano-PE, mais 151,4 km na 232 até Recife-PE, totalizando um percurso de $232 \mathrm{~km}$.

\subsection{Principais aspectos fisiográficos}

Possui um relevo ondulado em forma de colinas, como pode ser observado na Figura 3. Com cotas que podem chegar a $1.030 \mathrm{~m}$ (L 772937,56 m / S 9017673,73 $\mathrm{m})$, como no caso da superfície de cimeira do morro do Magano, a média altimétrica do município é $850 \mathrm{~m}$.

Mesmo estando situado no polígono das secas da região Nordeste, devido ao domínio do clima semiárido, apresenta-se como uma área atípica, ficando sob a influência do clima Mesotérmico Tropical de altitude.

Conforme o Instituto Nacional de Meteorologia INMET (2013), sua precipitação média anual é superior a $80 \mathrm{~mm}$, julho é o que apresenta a maior média 155,8 $\mathrm{mm}$, e o mês de novembro a menor $23 \mathrm{~mm}$. O período mais chuvoso vai de maio a agosto, com uma média pluviométrica de $132,2 \mathrm{~mm}$, já o quadrimestre com menores índices pluviométricos começa em setembro e termina em dezembro, com uma precipitação média de $34,7 \mathrm{~mm}$. Suas temperaturas são amenas, média anual de $21,6^{\circ} \mathrm{C}$, o período mais frio tem seu início em junho e o término em setembro, apresentando média de $19,6^{\circ} \mathrm{C}$, as temperaturas mais elevadas estão no quadrimestre dezembro/março, com média de $23,3^{\circ} \mathrm{c}$.

Originalmente possuía duas coberturas vegetais principais, mata atlântica na sua porção barlavento, e caatinga hipoxerófila a sotavento. Isso ocorre porque a referida área encontra-se situada na transição entre agreste e sertão. Mas atualmente só existem resquícios dessas vegetações.

Estando inserido na Bacia Hidrográfica do Mundaú. Essa rede hidrográfica fica localizada nos Estados de Pernambuco e Alagoas, abrangendo uma área de 4.090,39 $\mathrm{km}^{2}$ dos quais 2.154,26 km² estão em Pernambuco, correspondendo a $2.19 \%$ de sua área. A porção compreendida no território pernambucano encontra-se delimitada pelos paralelos $08^{\circ} 41^{\prime} 34^{\prime \prime} / 09^{\circ} 14^{\prime} 00^{\prime \prime} \mathrm{S}$ e pelos meridianos de $36^{\circ} 03^{\prime} 36^{\prime \prime} / 36^{\circ} 37^{\prime} 27^{\prime \prime} \mathrm{W}$.

\section{Materiais e métodos}

Foram usadas imagens oriundas do satélite Landsat 5, sensor TM (Thematic Mapper), com varredura de 185/185 Km, com resolução espacial de $30 \mathrm{~m}$, bandas: $1,0,45$ a $0,52 \mu \mathrm{m}$, azul; $2,0,52$ a $0,60 \mu \mathrm{m}$, verde; $3,0,63$ a 0,69 $\mu \mathrm{m}$, vermelho; $4,0,76$ a $0,90 \mu \mathrm{m}$, infravermelho próximo; $5,1,55$ a 1,75 $\mu \mathrm{m}$, infravermelho médio; $7,2,08$ a 2,35 $\mu \mathrm{m}$, infravermelho distante. Referentes aos intervalos temporais de 13/08/1987, 22/10/2001 e 29/09/2010.

Os Sistemas de Informações Geográficas (SIGs), utilizados para o tratamento/correções radiométricas e confecção da base de dados e mapas temáticos foram:

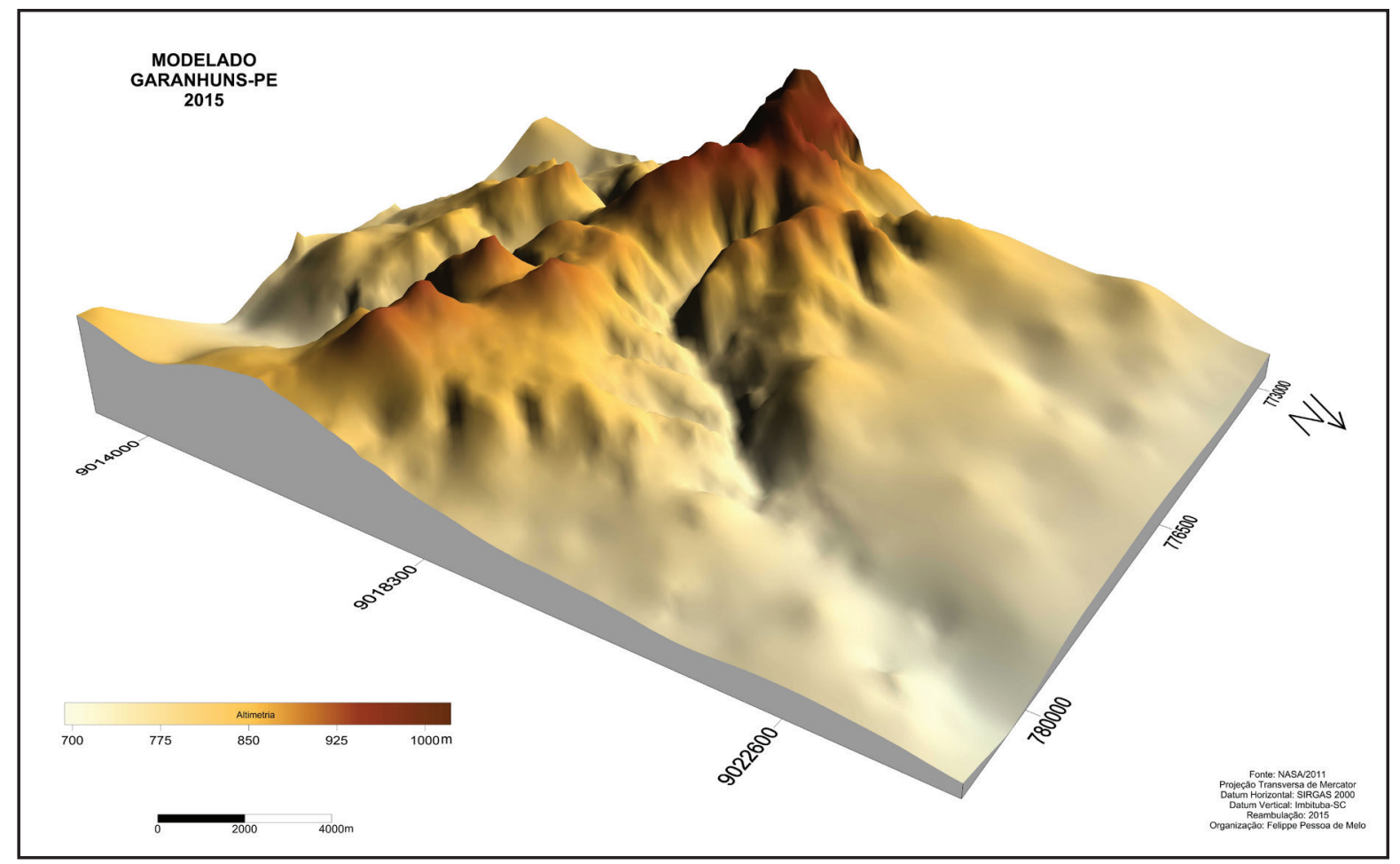

Figura 3 - 3D da área de estudo

Fonte: INPE (2011) 
TerraView, 4.2.2; Global Mapper, v. 15; SPRING, v. 5.2.7; ArcGIS, v. 10.2. Sendo assim, os procedimentos operacionais foram realizados em quatro etapas.

\subsection{Calibração radiométrica}

No TerraView, realizaram-se os procedimentos: confecção de banco de dados geográficos (Arquivo/Banco de Dados/Criar), importação dos rasters (Arquivo/ Importação Simples do Raster), correção das interferências atmosféricas (Plugins/Processamento de Imagem/ Funções/Restauração).

Dessa forma cada pixel das imagens primárias, são transformados em radiância espectral monocromática. Utilizando o método de Markham; Baker (1987), a partir da equação: $L K=a_{i}+\left(b_{i}-a_{i} / 255 * N D\right)$. De forma que (a) e (b), são as radiância espectrais mínimas e máximas $\left(\mathrm{W} \mathrm{m} \mathrm{m}^{-2} \mathrm{sr}^{-1} \mu \mathrm{m}^{-1}\right)$, detectada pelo sensor TM do satélite Landsat 5; ND, é a intensidade de cada pixel, com calor inicial de 0 e final de 255; i, corresponde aos canais com resolução espacial de $30 \mathrm{~m}$. Chander et al. (2007), propõe coeficientes de calibração distintos para radiância espectral, conforme Tabela1.

Allen et al. (2002 apud Machado, et al., 2007, p. 7358), a reflectância planetária é a razão entre a integração hemisférica da radiância monocromática incidente em um objeto. Sendo estimada pela equação: $\mathrm{P}_{\mathrm{Ki}}=\pi^{*} \mathrm{~L}_{\mathrm{Ki}} /$ ESUN ${ }^{*} \cos Z^{*} \mathrm{~d}_{\mathrm{r}}$ Onde $\mathrm{L}_{\mathrm{Ki}}$ é a radiância espectral de cada banda, ESUN $\kappa_{\kappa}$ é a irradiância solar espectral da cada banda no topo da atmosfera $\left(\mathrm{W} \mathrm{m}^{-2} \mu \mathrm{m}^{-1}\right)$, $\mathrm{Z}$ é o ângulo zenital solar e $\mathrm{d}_{\mathrm{r}}$ é o inverso do quadrado da distância relativa Terra/Sol.

\subsection{Correção de Datum}

As imagens disponibilizadas pelo INPE, originalmente apresentam-se com o datum WGS84, mas as normas brasileiras em vigor exigem que as informações geográficas utilizem o datum SIRGAS 2000. Para tanto utilizou-se o SIG Global Mapper, os procedimentos foram: importação das imagens (File/Oppen Datta File(s)), troca de datum (Tools/Configure/Projection) e exportação no formato Geotiff (File/Export/Export Raster - Image Format).

\subsection{Retificação do Georreferenciamento}

No software SPRING, foi elaborado um banco de dados geográficos (Arquivo/Banco de Dados) e um projeto (Arquivo/Projeto/Projetos). Em seguida as imagens pré-processadas no programa TerraView e no Global Mapper, foram abertas no módulo Impima (Arquivo/ abrir) e posteriormente exportadas (Arquivo/Salvar Como). A próxima etapa foi à importação das imagens (Arquivo/Importar/Importar Imagens Registradas); correção do georreferenciamento (Arquivo/Registro de Imagem/Teclado/Imagens); importação dos rasters retificados (Arquivo/Importar/Importar Imagens Registradas); composição colorida em falsa cor - b3 (b), b4 (g) e b5 (r), correção dos canais - tonalidades dos histogramas (Imagem/Contraste/Canal), confecção de imagem sintética (Imagem/Contraste/Sintética); exportação dos rasters no formato Geotiff (Arquivo/Exportar/Exportar Dados Vetoriais e Matriciais/Monocromático).

\subsection{Correção do albedo e confecção das cartas temáticas}

Conforme Allen et al. (2002 apud Moreira; Nóbrega, 2011, p. 0778), o computo do albedo planetário, isto é, o albedo não ajustado a transmissividade atmosférica, que é obtida pela combinação linear das refletâncias monocromáticas dos canais reflectivos do TM-Landsat 5. Sendo mensurado pela equação: $A_{\text {toa }}=0.293 * b 1+$ $0,274 * b 2+0,233 * b 3+0,157 * b 4+0,033^{*}$ b5 $5+0,011 * b 7$.

Sendo assim as imagens foram adicionadas no ambiente do SIG (Add Data). Para obtenção dos resultados da equação supracitada, utilizou-se a calculadora raster

Tabela 1 - Parâmetros para correção radiométrica de imagens Landsat 5

\begin{tabular}{|c|c|c|c|c|c|c|c|c|c|}
\hline \multicolumn{10}{|c|}{ 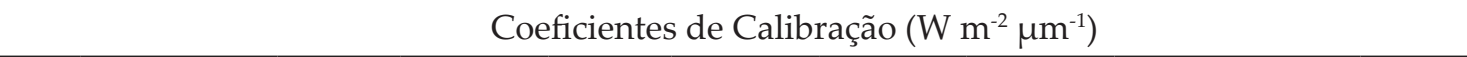 } \\
\hline$\frac{n}{\tilde{U}}$ & $\begin{array}{l}\text { Faixa } \\
\text { espectral } \\
\text { (um) }\end{array}$ & \multicolumn{2}{|c|}{$\begin{array}{c}01 / 03 / 1984 a \\
04 / 05 / 2003\end{array}$} & \multicolumn{2}{|c|}{$\begin{array}{c}05 / 05 / 2003 \text { a } \\
01 / 04 / 2007\end{array}$} & \multicolumn{2}{|c|}{$\begin{array}{l}\text { Posterior a } \\
01 / 04 / 2007\end{array}$} & \multirow[t]{2}{*}{ 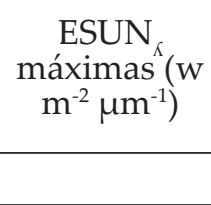 } & \multirow[t]{2}{*}{$W_{K i}$} \\
\hline & & $\mathrm{a}$ & $\mathrm{b}$ & a & $\mathrm{b}$ & a & $\mathrm{b}$ & & \\
\hline 1 & $0,45 / 0,52$ & $-1,52$ & 152,10 & $-1,52$ & 193 & $-1,52$ & 169 & 1957 & 0,293 \\
\hline 2 & $0,52 / 0,60$ & $-2,84$ & 296,81 & $-2,84$ & 365 & $-2,84$ & 333 & 1826 & 0,274 \\
\hline 3 & $0,63 / 0,69$ & $-1,17$ & 204,30 & $-1,17$ & 264 & $-1,17$ & 264 & 1554 & 0,233 \\
\hline 4 & $0,76 / 0,90$ & $-1,51$ & 206,20 & $-1,51$ & 221 & $-1,51$ & 221 & 1036 & 0,155 \\
\hline 5 & $1,55 / 1,75$ & $-0,37$ & 27,19 & $-0,37$ & 30,20 & $-0,37$ & 30.2 & 215 & 0,032 \\
\hline 7 & $2,08 / 2,35$ & $-0,15$ & 14,38 & $-0,15$ & 16,50 & $-0,15$ & 16.50 & 80,67 & 0,012 \\
\hline
\end{tabular}

Fonte: Chander et al., (2007). Adaptada pelos autores 
do ArcGIS (ArcToolbox/Spatial Analyst Tools/Map Algebra/Raster Calculator).

A obtenção do albedo da superfície foi feita pela equação: a (albedo da superfície $)=a_{\text {toa }}$ (albedo no topo da atmosfera) - ap (albedo planetário) $/ \mathrm{T}_{\mathrm{sw}}{ }^{2}$ (Transmissividade atmosférica que passa as condições que oscila de 0,025 a 0,04, (quanto melhores as condições atmosféricas, maior deve ser o valor) que é obtida pela equação: $T_{\mathrm{sw}}$ $=0,75+2 \cdot 10^{-5 *} \mathrm{Z}$ ). $Z$, é a média altimétrica dos pixels. Por fim foi realizado a confecção das cartas temáticas no módulo Layout View.

\section{Resultados e discussões}

De posse dos dados dos albedos dos respectivos intervalos temporais $(1987,2001$ e 2010), foram realizados os processos de interpretação e análise das imagens, com a finalidade de hierarquizar/padronizar as classes de uso e ocupação do solo, conforme a Tabela 2. Subsidiado pelo o método de Florenzano (2011), que toma como referência para análise das imagens: cor, textura, tamanho, forma, sombra, altura, padrão e localização.

Tabela 2 - Chave de interpretação das imagens

\begin{tabular}{|c|c|c|c|c|}
\hline \multirow[b]{2}{*}{$\begin{array}{c}\text { Elementos para a } \\
\text { Análise }\end{array}$} & \multicolumn{4}{|c|}{ Feições dos Alvos } \\
\hline & Vegetação & Solo Exposto & Perímetro Urbano & Nuvens \\
\hline Cor & Verde & Magenta ou Branca & Magenta - Predominante & Branca \\
\hline Textura & Rugosa & Lisa ou Rugosa & Rugosa & \\
\hline Tamanho & \multicolumn{4}{|c|}{ Variável } \\
\hline Forma & \multicolumn{4}{|c|}{ Irregular } \\
\hline Sombra & \multicolumn{4}{|c|}{ 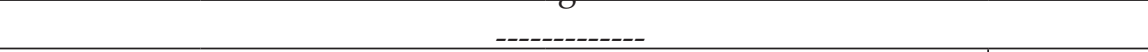 } \\
\hline Altura & \multicolumn{3}{|c|}{ - } & Variável \\
\hline Padrão & \multicolumn{4}{|c|}{ Irregular } \\
\hline Localização & \multicolumn{4}{|c|}{$\mathrm{S} 8^{\circ} 51^{\prime} 37^{\prime \prime} / \mathrm{S} 8^{\circ} 55^{\prime} 40^{\prime \prime}-\mathrm{O} 36^{\circ} 26^{\prime} 06^{\prime \prime} / \mathrm{O} 36^{\circ} 30^{\prime} 52^{\prime \prime}$} \\
\hline
\end{tabular}

Fontes: IBGE (2010); INPE(2015)

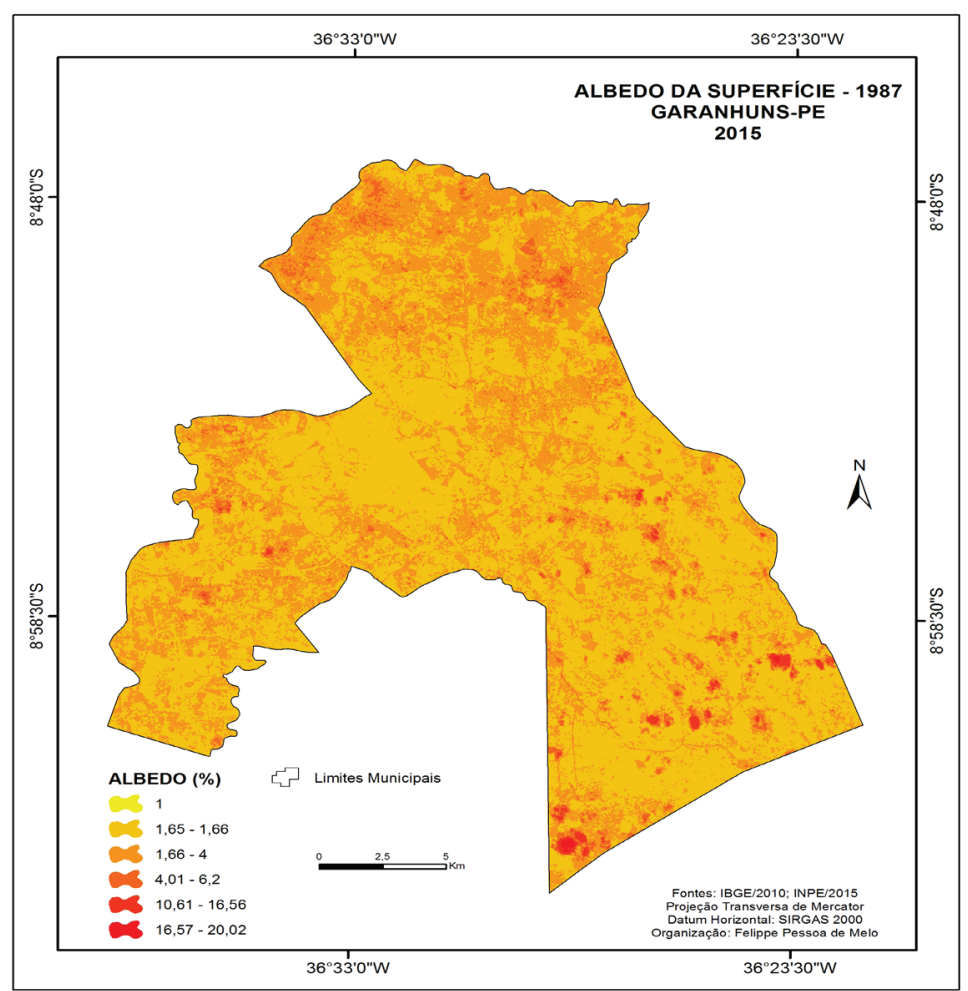

Figura 4 - Albedo de Garanhuns-PE em 1987

Fontes: IBGE (2010); INPE(2015) 
Os albedos de 1987, como pode ser analisado na Figura 4, apresentaram grandes amplitudes de reflectâncias, destacando-se os entre 1 e 1,66\%, disponíveis na Tabela 3.

Predominam nas classes: 1 e 2, vegetação mais densa; 3, cobertura vegetal dispersa e o sítio urbano a nordeste;
4, lotes residenciais no perímetro urbano e solos expostos nas demais áreas; 5 e 6, nuvens, do tipo cúmulos. Em 2001, conforme Figura 5, é fácil percepção a mudança no padrão do albedo da superfície municipal, sobressaindo-se a classe 2 (Tabela 4 ).

Tabela 3 - Dados estatísticos dos albedos de 1987

\begin{tabular}{c|c|c|c|c}
\hline Classe & Albedo $(\%)$ & Absorção $(\%)$ & No de Pixel & Área (ha) \\
\hline 1 & 1 & 99 & 43 & 3,87 \\
\hline 2 & $1,65-1,66$ & 98,33 & 311.381 & $28.022,9$ \\
\hline 3 & $1,66-4$ & 94,34 & 180.694 & $16.263,8$ \\
\hline 4 & $4,01-6,2$ & 89,79 & 15.259 & $1.373,31$ \\
\hline 5 & $10,61-16,56$ & 72,83 & 2.352 & 211,68 \\
\hline 6 & $16,57-20,02$ & 63,41 & 300 & 27 \\
\hline
\end{tabular}

Fontes: IBGE (2010); INPE(2015)

Tabela 4 - Dados estatísticos dos albedos de 2001

\begin{tabular}{c|c|c|cc}
\hline Classe & Albedo $(\%)$ & Absorção (\%) & No de Pixel & Área (ha) \\
\hline 1 & $0-2$ & 99 & 10 & 0,9 \\
\hline 2 & $2,21-4,94$ & 96,42 & 348.907 & $31.403,3$ \\
\hline 3 & $4,95-6,43$ & 94,31 & 133.849 & $12.044,6$ \\
\hline 4 & $6,44-9,5$ & 92,02 & 26.181 & $2.356,38$ \\
\hline 5 & $9,51-15,7$ & 87,04 & 1.076 & 96,84 \\
\hline 6 & $15,71-20,02$ & 82,13 & 6 & 0,54 \\
\hline
\end{tabular}

Fontes: IBGE (2010); INPE(2015)

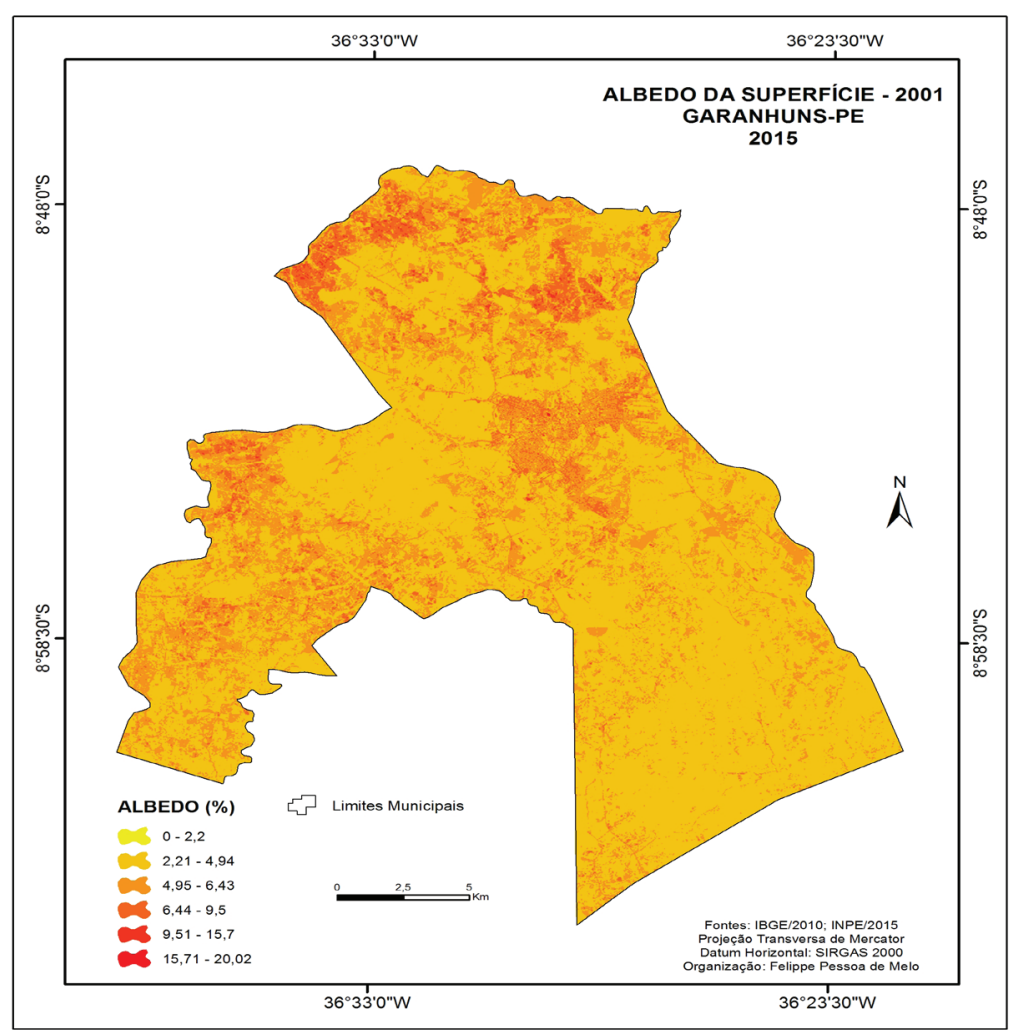

Figura 5 - Albedo de Garanhuns-PE em 2001

Fontes: IBGE (2010); INPE(2015) 
Comparando as médias de absorção de energia entre 1987 e 2001, observa-se que a primeira classe mantevese constante, mas reduziu a área; a segunda, diminuiu a absorção e a área; e terceira, teve resultados similares de absorção, mas apresentou uma maximização da poligonal; a quarta, minimizou a absorção e seu perímetro; a quinta, segue a tendência de aumento de reflectância, mas apresenta uma redução significativa de sua área; a sexta, reduz a absorção e maximiza de forma expressiva sua poligonal.

Tomando como parâmetro apenas o albedo observase que as classes 1 e 2 , onde encontra-se situado a maior porção do sítio urbano, apresentou uma maximização (média) do albedo, consequentemente uma redução da absorção de energia. Sendo que deve ser levado em consideração que, mesmo os alvos urbanos apresentando uma reflexão maior em relação aos naturais (na localidade em questão), eles têm uma capacidade maior de reter a energia absorvida. Variável essa que deve ser levada em consideração nos estudos termodinâmicos da superfície terrestre. Caso o contrário as análises indicarão que o aumento da mancha urbana maximiza o conforto térmico, já que ocorreu uma maximização da reflectância.

Sendo assim deve-se deixar bem claro/salientado que nem sempre o aumento da reflectância ou a minimização da absorção, significa melhor conforto térmico. A referida localidade de estudo é uma evidência dessa problemática

Tabela 5 - Dados estatísticos dos albedos de 2010

\begin{tabular}{c|c|c|cc}
\hline Classe & Albedo $(\%)$ & Absorção $(\%)$ & No de Pixel & Área (ha) \\
\hline 1 & $0-1,88$ & 99,06 & 7 & 0,63 \\
\hline 2 & $1,89-4,16$ & 96,97 & 228.688 & $20.584,3$ \\
\hline 3 & $4,17-5,02$ & 95,40 & 226.359 & $20.370,4$ \\
\hline 4 & $5,03-6,59$ & 94,19 & 47.759 & $4.297,86$ \\
\hline 5 & $6,6-13,81$ & 89,79 & 7.204 & 648,36 \\
\hline 6 & $13,82-20,02$ & 83,08 & 12 & 1,08 \\
\hline
\end{tabular}

Fontes: IBGE (2010); INPE(2015)

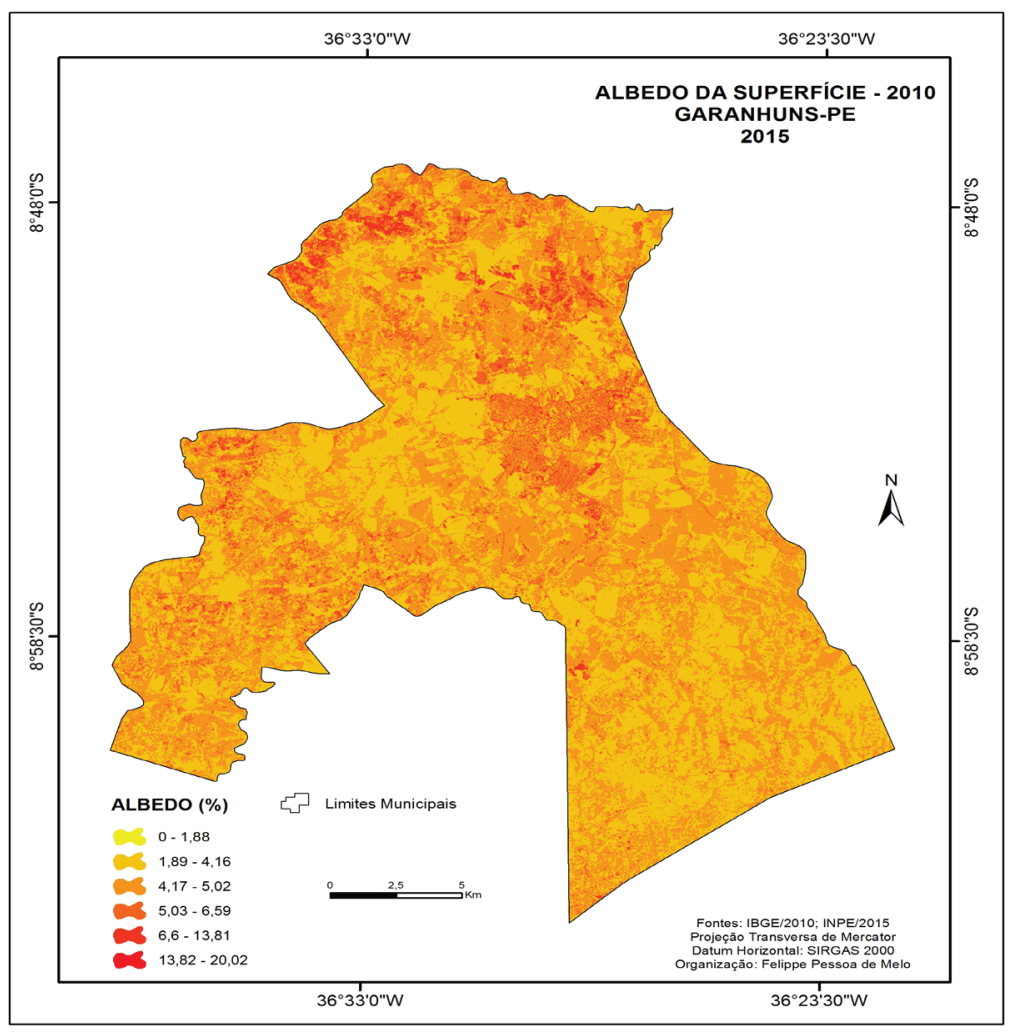

Figura 6 - Albedo de Garanhuns-PE em 2010

Fontes: IBGE (2010); INPE(2015) 
Em relação a reflectância, os dados apresentaram uma leve redução, de acordo com Tabela 5, sobressaindo-se a poligonal urbana, conforme a Figura 06. Compreendida entre a segunda e terceira classe. De forma geral, seguem as tendências dos anos pretéritos.

\section{Conclusões}

Diante dos resultados, ficou confirmado que com o transcorrer dos anos ocorreu o aumento do albedo, ou minimização da absorção, da energia eletromagnética, nos limites municipais.

Padrão esse que a princípio, indica um maior conforto termal. Mas quando levado em consideração que os objetos/alvos artificiais (de forma geral), apesar de possuírem uma maior capacidade de refletir a luz solar, em contra partida possuem o potencial de reter por mais tempo a energia absorvida.

Portanto o aumento do albedo na área em questão, não significou redução de temperaturas nas superfícies dos alvos. Dentro da poligonal municipal, o perímetro urbano sobressaiu-se, por apresentar as mudanças mais significativas no albedo.

Sendo assim, recomenda-se que nos estudos dos comportamentos dos alvos da superfície terrestre, seja levado em consideração que nem sempre o aumento do albedo é sinônimo de redução da temperatura das superfícies dos alvos. Principalmente quando a análise é multitemporal, ao passo que aumenta os intervalos, maximiza-se a necessidade de estudos sobre a capacidade dos alvos (artificiais) de reter energia, oriunda do espectro eletromagnético.

\section{Agradecimentos}

Ao Grupo de Pesquisa em Geoecologia e Planejamento Territorial - GEOPLAN, pelo incentivo a pesquisa.

\section{Referências}

ALLEN, R. G. et al. Surface energy balance algorithms for land - (SEBAL). Idaho implementation - Advanced training and users manual, v. 1, 2002. Disponível em: < file:///C:/Users/LAB\%20II\%20-\%2009/Downloads/sebal_ manual idho 2002.pdf>. Acesso em 10 out. 2014.

CHANDER, G. et al. Revised Landsat-5 Thematic Mapper Radiometric Calibration. IEEE Geosciene and Remote Sensing Letters, v.4, n. 3, p. 490-494, 2007.

CHRISTOFOLETTI, A. Modelagem de Sistemas Ambientais. 1ํa ed. São Paulo-SP: Blucher, 1999.

FLORENZANO, T. G. Iniciação em Sensoriamento
Remoto. $3^{\underline{a}}$ ed. São Paulo-SP: Oficina de Textos, 2011.

GALVÍNCIO, J. D. et al. Determinação do Albedo no Município de Belém do São Francisco, Com Base Em Imagens Landsat 7. Revista de Geografia do Programa de Pós-Graduação de Geografia da UFPE, v. 23, n. 3, p. 103-118, 2006. Disponível em: < http://www.revista.ufpe. br/revistageografia/index.php/revista/article/view/83/42>. Acesso em 12 jan. 2015.

IBGE - Instituto Brasileiro de Geografia e Estatística. Banco de Dados. Disponível em: <http://www.ibge. gov.br/home/default.php>. Acesso em 01 de jan. 2013.

INMET - Instituto Nacional de Meteorologia. Banco de Dados Meteorológicos para Ensino e Pesquisa. Disponível em: <http://www.inmet.gov.br/portal/index.php?r=bdmep/ $\underline{b d m e p}>$. Acesso em: 21 de dez. 2013.

INPE - Instituto Nacional de Pesquisas Espaciais. Banco de Dados Geomorfométricos do Brasil. Disponível em: http://www.dsr.inpe.br/topodata/>. Acesso em: 28 de dez. 2011.

MACHADO, C. C. C. Impacto da correção atmosférica no cômputo do albedo e do IVAS no PARNA Catimbau (PE - Brasil) utilizando imagens TM - Landsat 5 e o SEBAL/METRIC. Disponível em: $<$ http://www.dsr.inpe. br/sbsr2011/files/p0675.pdf >. Acesso em: 23 de jan. de 2014.

Markham, B. L.; Barker, J. L. Thematic mapper band pass solar exoatmospherical irradiances. International Journal of Remote Sensing, v. 8, n. 3, p. 517-523, 1987.

MENDONÇA, F.; DANNI-OLIVEIRA, I. M. Climatologia: noções básicas e climas do Brasil. $1^{\underline{a}}$ ed. São Paulo-SP: Oficina de Textos, 2007.

MOREIRA, E. B. M.; NÓBREGA, R. S. Uso de imagens multiespectrais aplicada à análise espaço-temporal dos padrões de Temperatura e albedo da superfície. Disponível em: <http://www.dsr.inpe.br/sbsr2011/files/ p1177.pdf>. Acesso em: 23 de jan. de 2014.

SILVA, B. B. et al. Determinação de Albedo de Áreas Irrigadas com Base em Imagens Landsat 5 - TM, Revista Brasileira de Agrometeorologia, v. 13, n. 2, p. 11-21, 2005. Disponível em: < http://www.dca.ufcg.edu.br/ DCA download/ISR/UFPE/Artigo\%20002-004\%20-\%20 Determina\%E7\%E30\%20Albedo.pdf>. Acesso em 14 jan. 2015. 